\title{
Comparison of the 7th and the 8th AJCC Staging System for Non-metastatic D2-Resected Lymph Node-Positive Gastric Cancer Treated with Different Adjuvant Protocols
}

\author{
Jeong II Yu, MD, PhD' \\ Do Hoon Lim, MD, PhD' \\ Jeeyun Lee, MD, PhD² \\ Won Ki Kang, MD, PhD² \\ Se Hoon Park, MD, PhD² \\ Joon Oh Park, MD, PhD² \\ Young Suk Park, MD, PhD² \\ Ho Yeong Lim, MD, PhD² \\ Seung Tae Kim, MD, PhD² \\ Su Jin Lee, MD, PhD² \\ Sung Kim, MD, PhD ${ }^{3}$ \\ Tae Sung Sohn, MD, PhD \\ Jun Ho Lee, MD, PhD ${ }^{3}$ \\ Ji Yeong An, MD, PhD 3 \\ Min Gew Choi, MD, PhD 3 \\ Jae Moon Bae, MD, PhD ${ }^{3}$ \\ Heejin Yoo, MS ${ }^{4}$ \\ Kyunga Kim, $\mathrm{PhD}^{4}$
}

Departments of ${ }^{1}$ Radiation Oncology, ${ }^{2}$ Internal Medicine, and ${ }^{3}$ Surgery, Samsung Medical Center, Sungkyunkwan University School of Medicine, Seoul, ${ }^{4}$ Statistics and Data Center,

Samsung Medical Center, Seoul, Korea

Correspondence: Do Hoon Lim, MD, PhD Department of Radiation Oncology,

Samsung Medical Center, Sungkyunkwan University School of Medicine, 81 Irwon-ro, Gangnam-gu, Seoul 06351, Korea

Tel: 82-2-3410-2612

Fax: 82-2-3410-2619

E-mail: dh8.lim@samsung.com

Received July 12, 2018

Accepted September 27, 2018

Published Online October 1, 2018

\section{Purpose}

The purpose of this study was to compare prognostic differentiation performances of the 7th and the 8th edition of American Joint Committee on Cancer (AJCC) staging system for gastric cancer (GC) patients.

\section{Materials and Methods}

A total of 1,633 GC patients who underwent curative D2 resection followed by adjuvant chemotherapy alone (CA) or concurrent chemo-radiotherapy (CCRT) from 2004 to 2013 were included. Concordance index (c-index) was applied to compare the discriminatory ability.

\section{Results}

In the 8th edition, migration of stage was detected in 248 patients (15.2\%). Among them, 121 patients were up-staged while 127 patients were down-staged. Overall, there was no statistically significant difference in the discriminatory ability between the 7th and 8th editions. The new edition of staging system, however, showed a trend of better prognostic performance not only in recurrence-free survival (c-index $=0.734 ; 95 \%$ confidence interval $[\mathrm{Cl}]$, 0.706 to 0.762 in the 7 th edition vs. $c$-index $=0.740 ; 95 \% \mathrm{Cl}, 0.712$ to 0.768 in the 8 th edition; $p=0.14$ ), but also in overall survival (c-index $=0.717 ; 95 \% \mathrm{Cl}, 0.688$ to 0.745 in the 7 th edition vs. $c$-index $=0.722 ; 95 \% \mathrm{Cl}, 0.694$ to 0.751 in the 8 th edition; $p=0.19$ ), especially in stage III. This finding was repeated in the subgroup analysis regardless of adjuvant CA or CCRT.

\section{Conclusion}

Generally, the 8th edition of AJCC staging system had failed to show a superior discriminatory ability for curatively D2 resected GC patients than the 7th edition, although there was a trend of better prognostic performance of the new edition, regardless of adjuvant treatment method. 


\section{Introduction}

Stomach cancer is the second most common malignant tumor and the third most common cause of cancer-related death in Korea [1]. Although its incidence has been decreasing, stomach cancer remains one of the most common causes of cancer-related mortality worldwide [2,3].

Like other malignancies, tumor $(\mathrm{T})$, node $(\mathrm{N})$, metastasis (M) staging system for stomach cancer developed by the American Joint Committee on Cancer (AJCC) is most widely accepted and commonly used worldwide to predict prognosis and determine treatment strategy $[4,5]$. Recently, the new 8th edition of AJCC TNM staging system has been implemented in oncologic fields, including the management of stomach cancer [6].

Although this new version of the AJCC staging system has been validated with several retrospective cohorts [7-11], its validation in group treated homogeneously including D2 resection and adjuvant treatment has not been reported, particularly in those receiving adjuvant concurrent chemoradiotherapy (CCRT).

Based on such background, we performed this retrospective study to compare the 7th and the 8th editions of AJCC TNM staging system for stomach cancer patients with nonmetastatic D2-resected pathologic lymph node (LN) metastasis who received adjuvant chemotherapy or CCRT.

\section{Materials and Methods}

\section{Patients}

From institutional registry database, data of stomach cancer patients who had received curative D2, R0 resection with LN metastasis confirmed pathologically at Samsung Medical Center from December 2004 to January 2013 were collected. Patients were excluded in the present study according to the following criteria: (1) distant metastasis or peritoneal seeding on or before surgery; (2) coexisting or newly diagnosed malignancies within one year from the date of stomach cancer operation; (3) patients who did not receive adjuvant treatment or could not confirm whether adjuvant treatment was administered, or (4) patients who showed recurrence or had follow-up loss within two months from the date of operation.

Efficacy evaluation of adjuvant CCRT compared to adjuvant chemotherapy alone (CA) with the same study population group has been reported in another publication [12].

\section{Adjuvant treatment}

ARTIST (Adjuvant Chemoradiation Therapy in Stomach Cancer) trial, a randomized phase III study comparing adjuvant CCRT with CA in D2 resected stomach cancer, was conducted during the period of this study. All eligible patients were recommended to participate in this trial. Patients consented to enroll in ARTIST trial received CCRT (two cycles of capecitabine and cisplatin [XP] followed by 45 Gy of external beam radiotherapy (RT) in 25 fractions with capecitabine and then two additional cycles of XP) or six cycles of XP according to the protocol based on randomization [13]. If patient was ineligible or refused to enroll in the ARTIST trial, we recommended adjuvant CCRT according to INT-0116 protocol (one cycle of fluorouracil and leucovorin [FL] followed by 45 Gy of external beam RT in 25 fractions with FL) $[14,15]$. Details of radiotherapy are described in the other publication.

After outcomes of randomized phase III trials demonstrating the superiority of adjuvant chemotherapy in Korean and Japanese, S-1 or capecitabine and oxaliplatin (XELOX) were also used as valuable options as adjuvant treatment for stomach cancer [16,17]. The decision of adjuvant CCRT or CA was made by the patient after obtaining sufficient explanation from the medical oncologist.

\section{Follow-up}

Adjuvant treatment with either CA or CCRT was determined by the medical oncologist with full informed consent of patients at 4 to 6 weeks after the surgery. After completion of adjuvant treatment, regular follow-up was conducted every 3 months in the first year, every 6 months during the next 2 years, and then yearly thereafter. Recurrence of disease was diagnosed with pathologic examination as much as possible or with radiologic examinations.

\section{Statistical analysis}

All enrolled patients were staged using the 7 th and the 8 th staging system for analysis. Recurrence-free survival (RFS) and overall survival (OS) were calculated from the date of operation to the date of event (recurrence and death, respectively) detection or the last follow-up. They were estimated using the Kaplan-Meier method and compared using the logrank test. The significance of survival prediction for each staging system was assessed using Cox proportional-hazards regression analysis. Harrell's concordance index (c-index), a common measure of predictive power [18], was applied to compare two prognostic systems using a package compare in R ver. 3.4.3 (Vienna, Austria; http:// www.R-project.org/). All statistical analyses were performed using SAS ver. 9.4 
(SAS Institute Inc., Cary, NC) and R 3.4.3. Statistical significance was considered at $\mathrm{p}<0.05$.

\section{Ethical statement}

The present study was approved by the Institutional Review Board of Samsung Medical Center (IRB No. 2018-06113). The necessity of written informed consent was waived due to its retrospective nature.

\section{Results}

\section{Patients}

Among 11,714 patients operated and registered in Samsung Medical Center registry of stomach cancer from December 2004 to January 2013, a total of 1,633 patients with D2 resected and pathologic LN metastasis (909 patients treated with adjuvant CCRT and 724 patients treated with adjuvant CA) who satisfied the eligibility of the present study were finally enrolled. Details of the information about patient selection were reported in the previous study [12]. The median follow-up of all patients was 65.4 months (range, 3.9 to 141.7 months).

Baseline characteristics of all enrolled patients are summarized in Table 1. Median age of these patients was 55 years (range, 22 to 84 years). About $35 \%$ of all patients received total gastrectomy. Median numbers of dissected and metastatic LN were 44 (range, 15 to 142) and 4 (range, 1 to 54), respectively. Specific baseline characteristics according to adjuvant CCRT or CA are presented in S1 Table. Between the two groups, there was a significant difference in $\mathrm{N}$ category in contrast to $\mathrm{T}$ category.

\section{Comparison of stage difference between the 7th and the 8th edition}

The distribution of patients according to each edition of staging system is shown in Fig. 1. Six of 371 stage IIB patients $(1.6 \%)$ and 40 of 360 stage IIIA patients $(11.1 \%)$ according to the 7th edition of AJCC staging system were reclassified as IIIB stage according to the 8th edition of AJCC staging system. Of 298 patients with stage IIIB based on the 7th edition, 75 patients $(25.2 \%)$ were changed to stage IIIC while 60 patients $(20.1 \%)$ were changed to stage IIIA in the new staging system (the 8th edition). In addition, 67 of 115 patients $(58.2 \%)$ with stage IIIC previously were redistributed to stage IIIB in the 8th edition of the staging system. Outcomes of stage redistribution by the 7th and the 8th AJCC staging sys-
Table 1. Baseline characteristics of all enrolled patients

\begin{tabular}{|c|c|}
\hline Variable & No. of patients (\%) \\
\hline Age $(y r)$ & $55(22-84)$ \\
\hline \multicolumn{2}{|l|}{ Sex } \\
\hline Male & $1,056(64.7)$ \\
\hline Female & $577(35.3)$ \\
\hline \multicolumn{2}{|l|}{ Type of operation } \\
\hline Total & $571(35.0)$ \\
\hline Subtotal & $1,062(65.0)$ \\
\hline \multicolumn{2}{|c|}{ Classification of Lauren } \\
\hline Intestinal & $550(33.7)$ \\
\hline Diffuse & 815 (49.9) \\
\hline Mixed & $15(0.9)$ \\
\hline Unclassified & $253(15.5)$ \\
\hline No. of dissected LN & $44(15-142)$ \\
\hline No. of LN metastases & $4(1-54)$ \\
\hline \multicolumn{2}{|l|}{ T category } \\
\hline $\mathrm{T} 1$ & 256 (15.7) \\
\hline T2 & $698(42.7)$ \\
\hline T3 & 465 (28.5) \\
\hline T4a & $197(12.1)$ \\
\hline $\mathrm{T} 4 \mathrm{~b}$ & $17(1.0)$ \\
\hline \multicolumn{2}{|l|}{$\mathrm{N}$ category } \\
\hline N1 & $556(34.0)$ \\
\hline N2 & $500(30.6)$ \\
\hline N3a & $257(25.4)$ \\
\hline N3b & $120(10.0)$ \\
\hline \multicolumn{2}{|l|}{ Lymphatic invasion } \\
\hline Yes & $1,212(74.2)$ \\
\hline No & $421(25.8)$ \\
\hline \multicolumn{2}{|l|}{ Venous invasion } \\
\hline Yes & $258(15.8)$ \\
\hline No & $1,375(84.2)$ \\
\hline \multicolumn{2}{|l|}{ Perineural invasion } \\
\hline Yes & $762(46.7)$ \\
\hline No & $871(53.3)$ \\
\hline \multicolumn{2}{|l|}{ Adjuvant protocol } \\
\hline INT-0116 & $836^{\text {a) }}(51.2)$ \\
\hline ARTIST & $272(16.7)$ \\
\hline ACT-GC & 489 (29.9) \\
\hline CLASSIC & $23(1.4)$ \\
\hline Other & $13^{\mathrm{b})}(0.8)$ \\
\hline
\end{tabular}

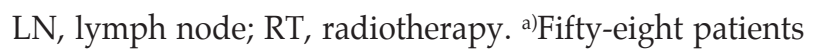
had received only 5 -fluorouracil (5-FU) and leucovorin chemotherapy according to the INT-0116 protocol without $\mathrm{RT},{ }^{\mathrm{b})}$ Other treatments used in 13 patients were as follows: TS-1 and capecitabine (6), tegafur/ uracil (2), capecitabine (1), TS-1/oxaliplatin (1), etoposide/ cisplatin (1), 5-FU/ epirubicin/cisplatin (1), and tremelimumab (1) were used in these patients. 


\begin{tabular}{|c|c|c|c|c|c|}
\hline \multicolumn{4}{|c|}{ 7th ed. } & \multicolumn{2}{|c|}{ 8th ed. } \\
\hline IB & 146 & T1N1 & 146 & IB & 146 \\
\hline \multirow[t]{2}{*}{$\| \mathrm{A}$} & 343 & T1N2 & 78 & $\| A$ & 343 \\
\hline & & T2N1 & 265 & & \\
\hline \multirow[t]{4}{*}{$\| B$} & 371 & T1N3a & 26 & IIB & 365 \\
\hline & & T1N3b & 6 & & \\
\hline & & T2N2 & 233 & & \\
\hline & & T3N1 & 106 & & \\
\hline \multirow[t]{4}{*}{$\| \mathrm{A} A$} & 360 & T2N3a & 160 & IIIA & 380 \\
\hline & & T2N3b & 40 & & \\
\hline & & T3N2 & 125 & & \\
\hline & & T4aN1 & 35 & & \\
\hline \multirow[t]{4}{*}{ IIIB } & 298 & T3N3a & 159 & IIIB & 276 \\
\hline & & T3N3b & 75 & & \\
\hline & & T4aN2 & $60^{\circ}$ & & \\
\hline & & T4bN1 & 4 & & \\
\hline \multirow[t]{5}{*}{ IIIC } & 115 & T4aN3a & $63^{\circ}$ & IIIC & 123 \\
\hline & & T4aN3b & 39 & & \\
\hline & & T4bN2 & 4 & & \\
\hline & & T4bN3a & 6 & & \\
\hline & & T4bN3b & 3 & & \\
\hline
\end{tabular}

Fig. 1. The number of patients redistributed in the 8th edition from the 7th edition of the American Joint Committee on Cancer (AJCC) staging system for gastric cancer is displayed. The solid line indicates upstaging while the dot line indicates downstaging.

tem for enrolled patients according to adjuvant treatment are presented in S2 Fig.

\section{Comparison of the performance in recurrence and RFS}

Table 2 shows results of crude recurrence rates for each stage classified according to the two staging systems. As a prognostic factor for recurrence, both the 7th and 8th staging systems showed statistically significant stratification ability in term of recurrence rate according to each stage (both $p<$ 0.001). Although the 8th AJCC staging system showed better discrimination ability between stage IIIA and IIIC, the most advanced stages, there was no statistically significant difference in prognosticator ability of recurrence rate between the two staging systems $(\mathrm{p}=0.14$; $\mathrm{c}$-index $0.734 ; 95 \%$ confidence interval [CI], 0.706 to 0.762 in 7 th vs. c-index $0.740 ; 95 \%$ CI, 0.712 to 0.768 in 8 th). The same tendency appeared in subgroup analysis depending on the presence of adjuvant CA ( $p=0.22$; c-index $0.722 ; 95 \% \mathrm{CI}, 0.680$ to 0.764 in 7 th vs. c-index $0.727 ; 95 \%$ CI, 0.685 to 0.769 in 8 th) or CCRT ( $p=0.26$; c-index $0.748 ; 95 \% \mathrm{CI}, 0.711$ to 0.785 in 7 th vs. c-index 0.755 ; $95 \%$ CI, 0.718 to 0.793 in 8th). There was no noticeable difference in discrimination ability between the 7 th and the 8 th staging systems in the subgroup of adjuvant CA as well as CCRT, although the new staging system showed significant difference in recurrence rate between stage IIIB and IIIC. Such difference was not detected in the old staging system.

Analysis of RFS reaffirmed those finding that there was no statistically significant difference, but more noticeable discrimination of survival curves for stage IIIA to IIIC in the 8th edition than that in the 7 th edition. This trend was identified regardless of adjuvant CA or CCRT treatment. Kaplan-Meier curves of RFS according to the 7th and the 8th AJCC staging system are presented in Fig. 2.

\section{Comparison of the performance in death and OS}

Of 404 patients $(24.7 \%)$ died during the follow-up, 165 $(22.8 \%)$ were adjuvant CA group patients and $239(23.3 \%)$ were adjuvant CCRT group patients. Crude death rate for each stage was classified according to the two staging systems. Results are shown in S3 Table.

As a prognostic factor for death, both the 7th and 8th staging systems showed statistically significant stratification ability according to each stage (both $\mathrm{p}<0.001$ ). Although there was no statistical difference in the prognosticator ability of 
Table 2. Comparison of recurrence rate prediction between the 7th and the 8th edition of AJCC staging system

\begin{tabular}{|c|c|c|c|c|c|c|c|c|}
\hline Group & System & Stage & $\begin{array}{c}\text { No. of } \\
\text { patients }\end{array}$ & $\begin{array}{c}\text { Recurrence, } \\
\text { n (\%) }\end{array}$ & & HR & $95 \% \mathrm{CI}$ & p-value \\
\hline \multirow[t]{12}{*}{ All } & \multirow[t]{6}{*}{ 7th } & IB & 146 & $3(2.1)$ & & & & $<0.001$ \\
\hline & & IIA & 343 & $30(8.7)$ & IB $^{\text {a) }}$ vs. IIA & 4.32 & $1.32-14.16$ & 0.020 \\
\hline & & IIB & 371 & $61(16.4)$ & IIA $^{\text {a) }}$ Vs. IIB & 2.09 & $1.35-3.24$ & $<0.001$ \\
\hline & & IIIA & 360 & $106(29.4)$ & IIB $^{\text {a) }}$ vs. IIIA & 1.91 & $1.39-2.62$ & $<0.001$ \\
\hline & & IIIB & 298 & $150(50.3)$ & IIIA $^{\text {a) }}$ vs. IIIB & 2.03 & $1.58-2.60$ & $<0.001$ \\
\hline & & IIIC & 115 & $69(60.0)$ & IIIB $^{\text {a) }}$ vs. IIIC & 1.38 & $1.04-1.84$ & 0.030 \\
\hline & \multirow[t]{6}{*}{ 8th } & IB & 146 & $3(2.1)$ & & & & $<0.001$ \\
\hline & & IIA & 343 & $30(8.7)$ & IB $^{\text {a) }}$ vs. IIA & 4.33 & $1.32-14.17$ & 0.020 \\
\hline & & IIB & 365 & $59(16.2)$ & IIA $^{a)}$ Vs. IIB & 2.05 & $1.32-3.18$ & 0.001 \\
\hline & & IIIA & 380 & $112(29.5)$ & IIB $^{\text {a) }}$ vs. IIIA & 1.95 & $1.42-2.67$ & $<0.001$ \\
\hline & & IIIB & 276 & $136(49.3)$ & IIIA $^{\text {a) }}$ vs. IIIB & 1.93 & $1.50-2.48$ & $<0.001$ \\
\hline & & IIIC & 123 & 79 (64.2) & IIIB $^{\text {a) }}$ vs. IIIC & 1.70 & $1.29-2.24$ & $<0.001$ \\
\hline \multirow[t]{12}{*}{ CA } & \multirow[t]{6}{*}{ 7th } & IB & 70 & $3(2.1)$ & & & & $<0.001$ \\
\hline & & IIA & 175 & $30(8.7)$ & IB $^{\text {a) }}$ vs. IIA & 2.20 & $0.64-7.53$ & 0.210 \\
\hline & & IIB & 184 & $59(16.2)$ & IIA $^{\text {a) }}$ Vs. IIB & 2.60 & $1.45-4.66$ & 0.001 \\
\hline & & IIIA & 135 & $112(29.5)$ & IIB $^{\text {a) }}$ vs. IIIA & 1.65 & $1.07-2.55$ & 0.020 \\
\hline & & IIIB & 111 & $136(49.3)$ & IIIA $^{\text {a) }}$ vs. IIIB & 1.66 & $1.11-2.48$ & 0.010 \\
\hline & & IIIC & 49 & 79 (64.2) & IIIB $^{\text {a) }}$ vs. IIIC & 1.56 & $1.00-2.44$ & 0.050 \\
\hline & \multirow[t]{6}{*}{ 8th } & IB & 70 & $3(4.3)$ & & & & $<0.001$ \\
\hline & & IIA & 175 & $16(9.1)$ & IB $^{\text {a) }}$ Vs. IIA & 2.20 & $0.64-7.54$ & 0.020 \\
\hline & & IIB & 183 & $37(20.2)$ & IIA $^{\text {a) }}$ vs. IIB & 2.54 & $1.41-4.56$ & 0.002 \\
\hline & & IIIA & 156 & $51(32.7)$ & IIB $^{\text {a) }}$ vs. IIIA & 1.73 & $1.13-2.64$ & 0.010 \\
\hline & & IIIB & 100 & $49(49.0)$ & IIIA $^{\text {a) }}$ vs. IIIB & 1.67 & $1.14-2.47$ & 0.010 \\
\hline & & IIIC & 40 & $27(67.5)$ & IIIB $^{\mathrm{a})}$ Vs. IIIC & 1.92 & $1.20-3.08$ & 0.007 \\
\hline \multirow[t]{12}{*}{ CCRT } & \multirow[t]{6}{*}{ 7th } & IB & 76 & 0 & & & & $<0.001$ \\
\hline & & IIA & 168 & $14(8.3)$ & IB $^{\text {a) }}$ Vs. IIA & 6.51 & $0.90-47.23$ & 0.060 \\
\hline & & IIB & 187 & $23(12.3)$ & IIA $^{\text {a) }}$ vs. IIB & 1.58 & $0.83-3.00$ & 0.160 \\
\hline & & IIIA & 225 & $63(28.0)$ & IIB $^{\text {a) }}$ vs. IIIA & 2.54 & $1.60-4.02$ & $<0.001$ \\
\hline & & IIIB & 187 & 97 (51.9) & IIIA $^{\text {a) }}$ vs. IIIB & 2.20 & $1.62-2.99$ & $<0.001$ \\
\hline & & IIIC & 66 & $39(59.1)$ & IIIB $^{\text {a) }}$ Vs. IIIC & 0.74 & $0.48-1.16$ & 0.190 \\
\hline & \multirow[t]{6}{*}{ 8th } & IB & 76 & 0 & & & & $<0.001$ \\
\hline & & IIA & 182 & $14(8.3)$ & IB $^{\text {a) }}$ Vs. IIA & 4.91 & $0.77-31.31$ & 0.090 \\
\hline & & IIB & 183 & $22(12.1)$ & IIA $^{\text {a) }}$ vs. IIB & 1.52 & $0.76-3.04$ & 0.023 \\
\hline & & IIIA & 224 & $61(27.2)$ & IIB $^{\text {a) }}$ vs. IIIA & 2.51 & $1.52-4.16$ & $<0.001$ \\
\hline & & IIIB & 176 & 87 (49.4) & IIIA $^{\text {a) }}$ vs. IIIB & 2.04 & $1.45-2.87$ & $<0.001$ \\
\hline & & IIIC & 83 & $52(62.7)$ & IIIB $^{\text {a) }}$ Vs. IIIC & 2.25 & $1.62-3.13$ & $<0.001$ \\
\hline
\end{tabular}

AJCC, American Joint Committee on Cancer; HR, hazard ratio; CI, confidence interval; CA, chemotherapy alone; CCRT, concurrent chemo-radiotherapy. ${ }^{\text {al }}$ Reference.

crude death rate between the two staging systems $(\mathrm{p}=0.19$; c-index $0.717 ; 95 \% \mathrm{CI}, 0.688$ to 0.745 in 7 th vs. c-index 0.722 ; $95 \% \mathrm{CI}, 0.694$ to 0.751 in 8 th), the 8 th edition generally showed better discrimination ability between stages IIB and IIIC than the 7th edition. Similar finding was also found for adjuvant $\mathrm{CA}$ systems ( $\mathrm{p}=0.22$; c-index $0.702 ; 95 \% \mathrm{CI}, 0.657$ to 0.746 in 7 th vs. c-index $0.708 ; 95 \%$ CI, 0.664 to 0.752 in 8 th) or CCRT ( $\mathrm{p}=0.49$; $\mathrm{c}$-index $0.727 ; 95 \% \mathrm{CI}, 0.689$ to 0.764 in 7 th vs. c-index $0.731 ; 95 \%$ CI, 0.694 to 0.768 in 8 th) subgroups (S4 Table).

In the analysis of OS, the 8th edition showed better discriminatory ability in OS curves, especially in advanced stages. This finding was also repeated regardless of adjuvant CA or CCRT treatment. Kaplan-Meier curves of OS according to the 7th and 8th AJCC staging systems are presented in Fig. 3. 

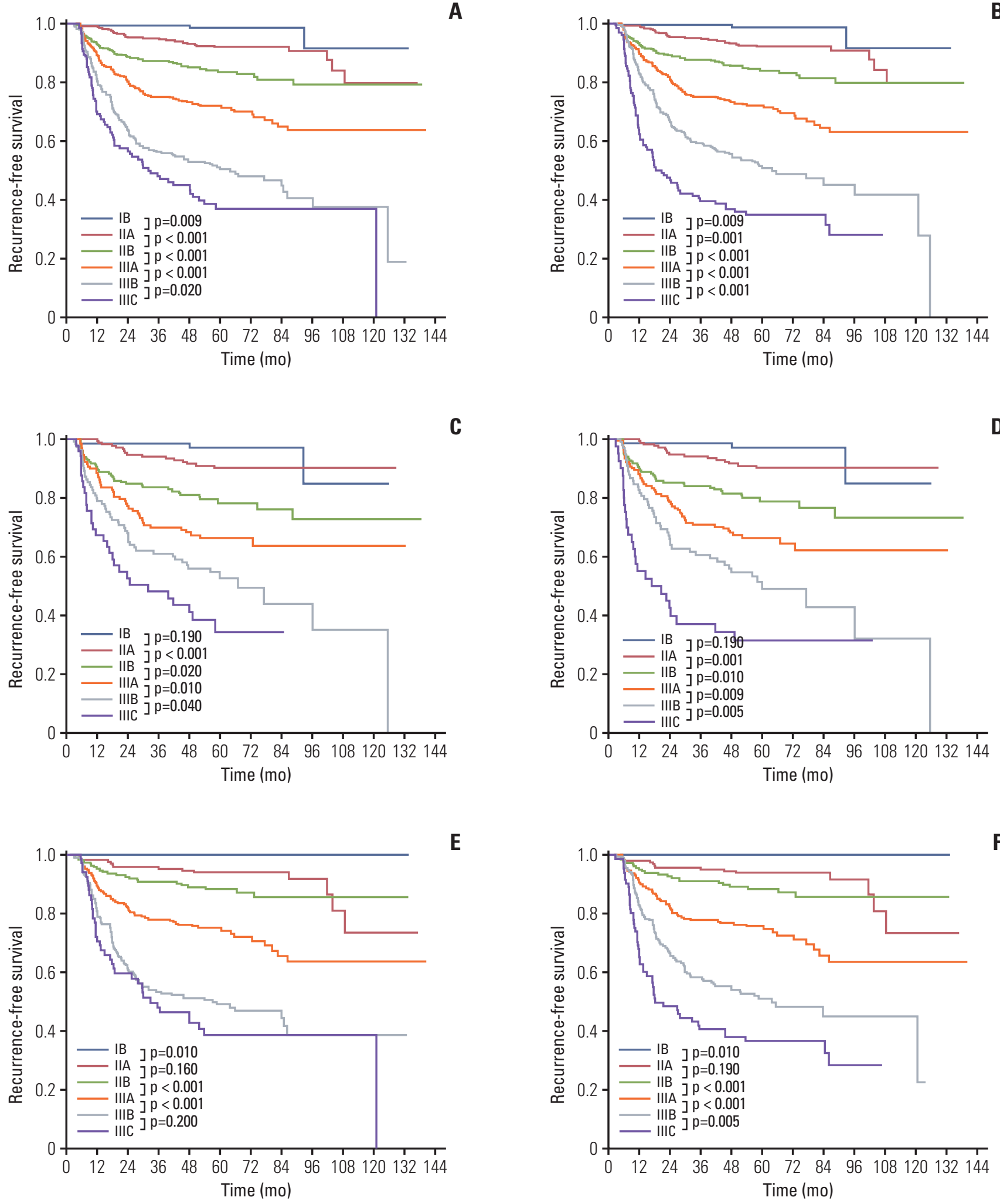

Fig. 2. Kaplan-Meier curves of recurrence-free survival according to the 7th versus the 8th edition of American Joint Committee on Cancer staging system. More clear discrimination of survival curves between stage IIIA to IIIC is shown in the 8th edition than that in the 7th edition for all (7th edition in A vs. 8th edition in B), CA (7th edition in C vs. 8th edition in D), and concurrent chemo-radiotherapy (7th edition in $\mathrm{E}$ vs. 8th edition in F) patients. 

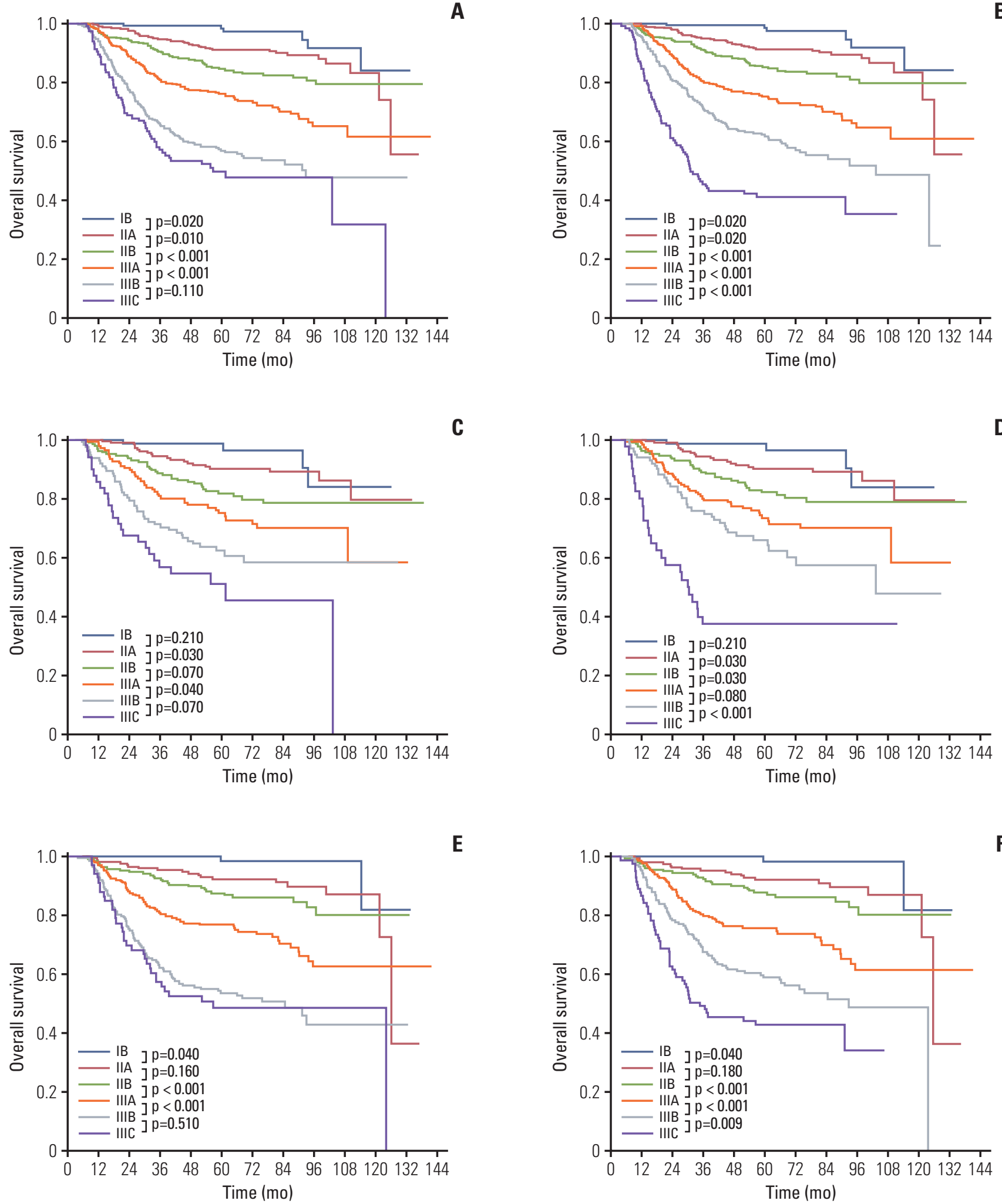

$\mathbf{E}$

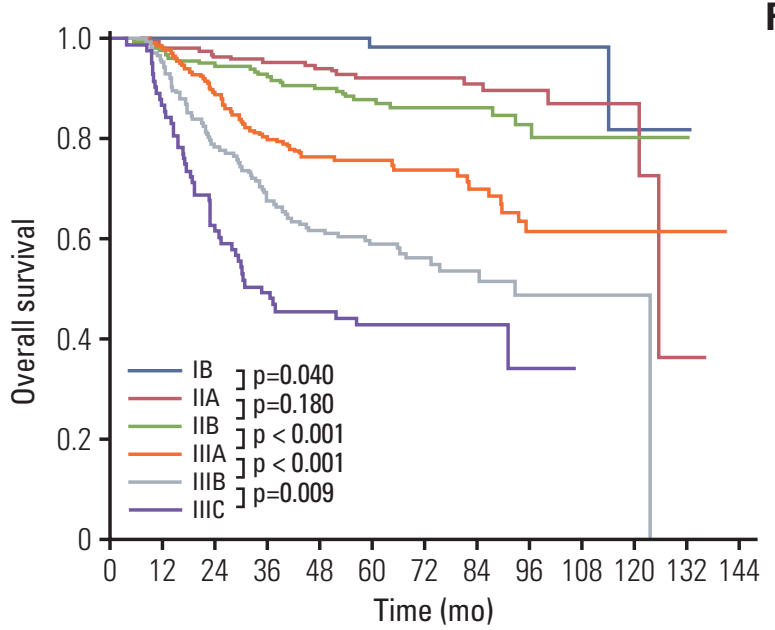

Fig. 3. Kaplan-Meier curves of overall survival according to the 7th versus the 8th edition of American Joint Committee on Cancer staging system. More clear discrimination of survival curves for stages IIIA to IIIC was shown in the 8th edition than that in the 7th edition for all (7th edition in A vs. 8th edition in B), chemotherapy alone (7th edition in C vs. 8th edition in D), and concurrent chemo-radiotherapy (7th edition in E vs. 8th edition in F) patients. 


\section{Discussion}

The present study was performed to evaluate whether the 8th edition was superior to the 7th edition of AJCC staging system in D2 resected gastric cancer patients with LN metastasis who received adjuvant CA or CCRT. Overall, the superiority of 8th edition than 7th edition was not identified in non-metastatic D2 resected gastric cancer (GC) patients treated with adjuvant CCRT or CA, both in the aspect of RFS as well as OS, though there was a trend of better prognostic performance in 8 th edition for advanced stage (IIIA to IIIC).

The presence of a widely accepted standardized staging system known to be strongly associated with prognosis is essential for proper management and research of tumor. Although several staging systems or prognostic factors have been suggested with the development of oncology [19-22], the AJCC staging system has been most widely accepted and used. In response to changes in tumor research and treatment techniques, the AJCC staging system is also undergoing constant modification. The 8 th edition of the AJCC staging system was announced in 2016 and started to be used in 2018.

Based on data of the International Gastric Cancer Association (IGCA), the 8th edition has been suggested to be superior to the 7th edition AJCC staging system in discriminatory ability for prognosis in terms of OS for each stage [23]. The performance of the 8th edition for prognostic separation is also clearly validated for U.S. population using National Cancer Database (NCDB) data [23]. This edition has also been successfully validated with large cohort of single Korean institution. It has been revealed that the new edition has superior prognostic discrimination for stage III disease [8].

It is well known that characteristics and prognosis of gastric cancer are clearly different between Eastern and Western populations [24]. Naturally, therapeutic approaches for Western and the Eastern populations remained controversial in despite of outcomes of several randomized phase III studies. In particular, the effect of D2 resection and the role of adjuvant RT after D2 resection remain debatable [14,25-28]. For this reason, adjuvant RT is rarely used in Eastern countries including Korea and Japan where D2 resection is accepted as a standard surgical procedure.

In our institution, D2 resection is performed as a standard procedure in stomach cancer. The ARTIST study, a randomized phase III trial to confirm the role of adjuvant RT after D2 resection was conducted by our institution $[13,29,30]$. In addition, ARTIST-II trial is ongoing based on possible beneficial effects of adjuvant RT in the subgroup of patients with LN metastasis in ARTIST study. Therefore, the cohort of our institution could be one of the best candidates to compare the performance of the 7th and the 8th AJCC staging systems, according to whether adjuvant CCRT or CA alone was used for non-metastatic $\mathrm{D} 2$ resected gastric cancer.

In this study, the 8th edition of AJCC staging system had failed to show a superior discrimination ability than the 7 th edition for non-metastatic D2 resected GC patients with LN metastasis treated with adjuvant CA and CCRT. The new staging system, however, showed higher prognostic discriminatory ability than the 7th edition regardless of whether adjuvant RT was used in stage III. In the new edition, the most important change in the staging system was that it emphasized the importance of the number of metastatic lymph nodes. N3b disease defined as 16 or more LNs metastases were upstaged in the new edition. Significant differences in prognosis according to LN stage including N3b stage have also been confirmed in our previous study based on the same cohort [12]. Based on the more distinct prognostic difference of the 8th edition of AJCC stating system, it could be possible to more accurately select management and outcome analysis in GC.

The present study has several limitations originating from the study design itself. First, this study was a retrospective single Korean tertiary referral institutional study. Enrolled patients from the Samsung Medical Center registry were not free from selection bias because they were confined to specific regions and characteristics among Korean. Second, caution is needed when generalizing outcomes of the present study because this study was performed only in Korean patients who underwent D2 resection, diagnosed with nonmetastatic GC with metastatic LN, and received adjuvant treatment. In particular, careful attention should be needed considering that the staging system should reflect the prognosis in all patients regardless of treatment. Third, this study did not analyze treatment outcome depending on adjuvant CA or CCRT according to the 8th edition of AJCC staging system. Despite those limitations, this study was performed in relatively large number of patients treated with current standard of care including D2 resection, adjuvant CA or CCRT, it could be informative and useful to predict or compare treatment outcomes in the same patient group receiving similar management.

In conclusion, the 8th edition of AJCC staging system had failed to show a superior discrimination ability than the 7th edition for non-metastatic D2 resected GC patients with LN metastasis treated with adjuvant CA and CCRT. However, the 8th edition demonstrated clear prognostic difference in stage III for both adjuvant treatment modalities, which difference was not detected in the 7th edition.

\section{Electronic Supplementary Material}

Supplementary materials are available at Cancer Research and Treatment website (https: // www.e-crt.org). 
Conflicts of Interest

Conflict of interest relevant to this article was not reported.

\section{Acknowledgments}

This research was partly supported by a Basic Science Research Program through the National Research Foundation of Korea (NRF) funded by the Ministry of Education (NRF-2017R1D1A1B03031275), and a grant from the Marine Biotechnology Program (20150220) funded by the Ministry of Oceans and Fisheries, Korea.

\section{References}

1. Jung KW, Won YJ, Kong HJ, Lee ES; Community of Population-Based Regional Cancer Registries. Cancer statistics in Korea: incidence, mortality, survival, and prevalence in 2015. Cancer Res Treat. 2018;50:303-16.

2. Global Burden of Disease Cancer Collaboration, Fitzmaurice C, Allen C, Barber RM, Barregard L, Bhutta ZA, et al. Global, regional, and national cancer incidence, mortality, years of life lost, years lived with disability, and disability-adjusted lifeyears for 32 cancer groups, 1990 to 2015: a systematic analysis for the global burden of disease study. JAMA Oncol. 2017;3: $524-48$.

3. Torre LA, Siegel RL, Ward EM, Jemal A. Global cancer incidence and mortality rates and trends: an update. Cancer Epidemiol Biomarkers Prev. 2016;25:16-27.

4. Kwon OK, Kim SW, Chae HD, Ryu SW, Chung HY, Kim SW, et al. Validation of the 7th AJCC/UICC staging system for gastric cancer and a proposal for a new TNM system based on a prognostic score: a retrospective multicenter study. Ann Surg Treat Res. 2016;91:295-302.

5. Shu P, Qin J, Shen K, Chen W, Liu F, Fang Y, et al. The IGCA staging system is more accurate than AJCC7 system in stratifying survival of patients with gastric cancer in stage III. BMC Cancer. 2017;17:238.

6. In H, Ravetch E, Langdon-Embry M, Palis B, Ajani JA, Hofstetter WL, et al. The newly proposed clinical and post-neoadjuvant treatment staging classifications for gastric adenocarcinoma for the American Joint Committee on Cancer (AJCC) staging. Gastric Cancer. 2018;21:1-9.

7. He X, Wu W, Lin Z, Ding Y, Si J, Sun LM. Validation of the American Joint Committee on Cancer (AJCC) 8th edition stage system for gastric cancer patients: a population-based analysis. Gastric Cancer. 2018;21:391-400.

8. Kim SG, Seo HS, Lee HH, Song KY, Park CH. Comparison of the differences in survival rates between the 7th and 8th editions of the AJCC TNM staging system for gastric adenocarcinoma: a single-institution study of 5,507 patients in Korea. J Gastric Cancer. 2017;17:212-9.

9. Li Z, Wang Y, Shan F, Ying X, Wu Z, Xue K, et al. ypTNM staging after neoadjuvant chemotherapy in the Chinese gastric cancer population: an evaluation on the prognostic value of the AJCC eighth edition cancer staging system. Gastric Cancer. 2018;21:977-87.
10. Lu J, Zheng CH, Cao LL, Li P, Xie JW, Wang JB, et al. The effectiveness of the 8th American Joint Committee on Cancer TNM classification in the prognosis evaluation of gastric cancer patients: a comparative study between the 7th and 8th editions. Eur J Surg Oncol. 2017;43:2349-56.

11. Lu J, Zheng ZF, Xie JW, Wang JB, Lin JX, Chen QY, et al. Is the 8th edition of the AJCC TNM staging system sufficiently reasonable for all patients with noncardia gastric cancer? A 12,549-patient international database study. Ann Surg Oncol. 2018;25:2002-11.

12. Yu JI, Lim DH, Lee J, Kang WK, Park SH, Park JO, et al. Necessity of adjuvant concurrent chemo-radiotherapy in D2resected LN-positive gastric cancer. Radiother Oncol. 2018;129: 306-12.

13. Lee J, Lim DH, Kim S, Park SH, Park JO, Park YS, et al. Phase III trial comparing capecitabine plus cisplatin versus capecitabine plus cisplatin with concurrent capecitabine radiotherapy in completely resected gastric cancer with D2 lymph node dissection: the ARTIST trial. J Clin Oncol. 2012;30:268-73.

14. Macdonald JS, Smalley SR, Benedetti J, Hundahl SA, Estes NC, Stemmermann GN, et al. Chemoradiotherapy after surgery compared with surgery alone for adenocarcinoma of the stomach or gastroesophageal junction. N Engl J Med. 2001;345:72530.

15. Smalley SR, Benedetti JK, Haller DG, Hundahl SA, Estes NC, Ajani JA, et al. Updated analysis of SWOG-directed intergroup study 0116: a phase III trial of adjuvant radiochemotherapy versus observation after curative gastric cancer resection. J Clin Oncol. 2012;30:2327-33.

16. Bang YJ, Kim YW, Yang HK, Chung HC, Park YK, Lee KH, et al. Adjuvant capecitabine and oxaliplatin for gastric cancer after D2 gastrectomy (CLASSIC): a phase 3 open-label, randomised controlled trial. Lancet. 2012;379:315-21.

17. Sakuramoto S, Sasako M, Yamaguchi T, Kinoshita T, Fujii M, Nashimoto A, et al. Adjuvant chemotherapy for gastric cancer with S-1, an oral fluoropyrimidine. N Engl J Med. 2007;357: 1810-20.

18. Harrell FE Jr, Lee KL, Califf RM, Pryor DB, Rosati RA. Regression modelling strategies for improved prognostic prediction. Stat Med. 1984;3:143-52.

19. Astler VB, Coller FA. The prognostic significance of direct extension of carcinoma of the colon and rectum. Ann Surg. 
1954;139:846-52.

20. Aurello P, D'Angelo F, Rossi S, Bellagamba R, Cicchini C, Nigri $G$, et al. Classification of lymph node metastases from gastric cancer: comparison between $\mathrm{N}$-site and $\mathrm{N}$-number systems. Our experience and review of the literature. Am Surg. 2007;73:359-66.

21. Chen XL, Zhao LY, Xue L, Xu YH, Zhang WH, Liu K, et al. Prognostic significance and the role in TNM stage of extranodal metastasis within regional lymph nodes station in gastric carcinoma. Oncotarget. 2016;7:67047-60.

22. Yu JI, Park HC, Lim DH, Park W, Yoo BC, Paik SW, et al. Prognostic index for portal vein tumor thrombosis in patients with hepatocellular carcinoma treated with radiation therapy. J Korean Med Sci. 2011;26:1014-22.

23. Amin MB, Edge S, Greene F, Byrd DR, Brookland RK, Washington MK, et al. AJCC cancer staing manual. 8th ed. New York: Springer; 2017.

24. Shim JH, Song KY, Jeon HM, Park CH, Jacks LM, Gonen M, et al. Is gastric cancer different in Korea and the United States? Impact of tumor location on prognosis. Ann Surg Oncol. 2014;21:2332-9.

25. GASTRIC (Global Advanced/Adjuvant Stomach Tumor Research International Collaboration) Group, Paoletti X, Oba K, Burzykowski T, Michiels S, Ohashi Y, et al. Benefit of adju- vant chemotherapy for resectable gastric cancer: a meta-analysis. JAMA. 2010;303: 1729-37.

26. Songun I, Putter H, Kranenbarg EM, Sasako M, van de Velde CJ. Surgical treatment of gastric cancer: 15-year follow-up results of the randomised nationwide Dutch D1D2 trial. Lancet Oncol. 2010;11:439-49.

27. Ohri N, Garg MK, Aparo S, Kaubisch A, Tome W, Kennedy TJ, et al. Who benefits from adjuvant radiation therapy for gastric cancer? A meta-analysis. Int J Radiat Oncol Biol Phys. 2013;86:330-5.

28. Dai Q, Jiang L, Lin RJ, Wei KK, Gan LL, Deng CH, et al. Adjuvant chemoradiotherapy versus chemotherapy for gastric cancer: a meta-analysis of randomized controlled trials. J Surg Oncol. 2015;111:277-84.

29. Park SH, Sohn TS, Lee J, Lim DH, Hong ME, Kim KM, et al. Phase III trial to compare adjuvant chemotherapy with capecitabine and cisplatin versus concurrent chemoradiotherapy in gastric cancer: final report of the adjuvant chemoradiotherapy in stomach tumors trial, including survival and subset analyses. J Clin Oncol. 2015;33:3130-6.

30. Yu JI, Lim DH, Ahn YC, Lee J, Kang WK, Park SH, et al. Effects of adjuvant radiotherapy on completely resected gastric cancer: a radiation oncologist's view of the ARTIST randomized phase III trial. Radiother Oncol. 2015;117:171-7. 